\title{
Características Biométricas Testiculares para Avaliação de Touros Zebuínos da Raça Nelore
}

\author{
Maria Marina Unanian¹, Antonio Emidio Dias Feliciano Silva1, Concepta McManus², Eduardo \\ Penteado Cardoso ${ }^{3}$
}

RESUMO - O objetivo deste estudo foi analisar as características de volume e forma testicular em zebuínos jovens da raça Nelore para caracterizar a sua importância na avaliação e seleção de reprodutores. Foram examinados a circunferência escrotal (CE), o comprimento (COMP), a largura (LARG) e o volume testicular (VOL) em 442 machos aos 12 e 18 meses de idade, divididos em dois grupos contemporâneos (G1 e G2), em função da data do nascimento. O VOL foi calculado pela fórmula: VOL $=2\left[\left(\mathrm{r}^{2}\right) \times \Pi \times \mathrm{h}\right] \mathrm{em} \mathrm{que} \mathrm{r}=\mathrm{LARG} / 2$, $\mathrm{h}=$ COMP, e $\Pi=3,14$. Os valores médios de CE, COMP, LARG e VOL, aos 12 meses de idade, foram 184,89 $\pm 15,79 \mathrm{~mm}, 65,96 \pm 6,48 \mathrm{~mm}$, $32,58 \pm 4,63 \mathrm{~mm}$ e $113,80 \pm 42,03 \mathrm{~cm}^{3}$, e aos 18 meses, $236,81 \pm \mathrm{mm}, 79,31 \pm 11,16 \mathrm{~mm}, 42,92 \pm 5,83 \mathrm{~mm}$ e $239,26 \pm 90,56 \mathrm{~cm}^{3}$, respectivamente. Os parâmetros, aos 12 e 18 meses de idade, variaram em função dos grupos contemporâneos, sendo altamente correlacionados. A forma dos testículos foi avaliada pela razão LARG/COMP, resultando em cinco formas: longa (razão $\leq 0,5$ ), longa moderada (razão de 0,51 a 0,625), longa/oval (razão de 0,626 a 0,750), oval/esférica (razão de 0,751 a 0,875) e esférica (razão > 0,875). Em 87\% dos animais, os testículos apresentaram forma longa e, em $74 \%$, houve mudança da forma testicular com a idade. Os parâmetros estudados variaram em função da forma testicular, e o crescimento dos testículos ocorreu em largura. Os resultados sugerem que, ao selecionar reprodutores, principalmente jovens, deve-se considerar o volume testicular, além de possíveis mudanças da forma testicular.

Palavras-chave: forma testicular, Nelore, touro, volume testicular

\section{Testicular Biometrics Characteristics to Evaluate the Nellore Zebu Breed Bulls}

ABSTRACT - The objective of this study was to analyze the testicular volume and shape in young males of Nellore Zebu breed and their importance for the evaluation and selection of sires. Four hundred and forty two males, from 12 to 18 month of age, were examined for scrotal circumference (CE), length (COMP), width (LARG) and testicular volume (VOL). The animals were divided in two groups (G1 and G2) according to their date of birth. The VOL was calculated by the formula: VOL $=2\left[\left(\mathrm{r}^{2}\right) \mathrm{x} \Pi \mathrm{x} \mathrm{h}\right]$, where $\mathrm{r}, \mathrm{h}$ and $\Pi$ are $\mathrm{LARG} / 2$, length and 3.14, respectively. The mean values of CE, COMP, LARG and VOL at 12 month of age were $184.89 \pm 15.79 \mathrm{~mm}, 65.96 \pm 6.48 \mathrm{~mm}$, $2.58 \pm 4.63 \mathrm{~mm}$ and $113.80 \pm 42.03 \mathrm{~cm}^{3}$, and at 18 months of age were $236.81 \pm \mathrm{mm}, 79.31 \pm 11.16 \mathrm{~mm}, 42.92 \pm 5.83 \mathrm{~mm}$ and $239.26 \pm 90.56$ $\mathrm{cm}^{3}$, respectively. The parameters at 12 and 18 months of age were influenced by contemporary groups, being highly correlated. The testicular shape was considered as a length to width ratio, being recorded five forms: long (ratio $\leq .5)$, long moderate (.51 to .626 ratio), long/ ovoid (.626 to $.750 \mathrm{ratio}$ ), ovoid/spheroid (.751 to $.875 \mathrm{ratio}$ ) and spheroid (> $.875 \mathrm{ratio}) .87 \%$ of the animals presented the long shape and, in $74 \%$, the testicular shape changed with the age. All the parameters were influenced by testicular shape, and the testis grew in width. The results suggest that the selection of young bulls should consider the testicular volume besides the possible change in the testicular shape.

Key Words: testicular shape, Nellore, bulls, testicular volume

\section{Introdução}

Constam do processo de seleção de reprodutores várias características morfo-fisiológicas do aparelho reprodutor, como a circunferência ou o perímetro escrotal e, ainda, mais recentemente, a avaliação quanti-qualitativa do sêmen.

Na preocupação de aumentar a precisão da escolha de reprodutores, estão sendo introduzidos outros conceitos - o volume testicular (BAILEY et al.,
1996; UNANIAN e SILVA, 1997) e a forma dos testículos (BAILEY et al., 1998).

Os estudos de BAILEY et al. (1996) verificaram que somente a circunferência escrotal não constitui medida representativa da produção espermática e, portanto, do potencial reprodutivo dos machos. Segundo esses autores, os testículos mais longos, como freqüentemente encontrados nas raças zebuínas, apresentam maior superfície de contato com o meio ambiente, o que facilita a termoregulação; além disso,

\footnotetext{
1 Pesquisador da EMBRAPA - Cenargen, SAIN Parque Rural, Final Av. W5 Norte, 70770-900 Brasília, DF. E.mail:unaniam@cenargem.embrapa.br; emidio@cenargem.embrapa.br

2 Professor Adjunto, Universidade de Brasília, Faculdade de Agronomia e Medicina Veterinária, 70910-970,Brasília, DF. E.mail:concepta@unb.br ${ }^{3}$ Eng. Agrônomo, Fazenda Mundo Novo, Brotas, SP.
} 
a distribuição dos vasos sangüíneos e do tecido espermático é mais uniforme, melhorando quantiqualitativamente o sêmen. Ainda, os mesmos autores observaram que os testículos de forma longa apresentaram volumes semelhantes às demais formas testiculares. Das pesquisas mencionadas, concluiuse que as formas testiculares mais alongadas apresentaram vantagens morfo-fisiológicas, sendo apontadas como favoráveis à reprodução.

Em função destas observações e considerando a forma testicular dos zebuínos, normalmente alongada, acredita-se que esta característica pode ter grande valor, principalmente, considerando as condições de temperatura em que vivem estes animais. Para testar estas hipóteses, conduziu-se este estudo para analisar a importância das características de forma e volume testicular, oferecer subsídios à seleção de reprodutores e conhecer melhor as características das raças zebuínas.

\section{Material e Métodos}

O estudo foi realizado em 442 machos da raça Nelore - PO, pertencentes a uma fazenda no município de Brotas - SP, que faziam parte da prova oficial de ganho de peso a pasto da ABCZ.Os animais deste experimento foram criados em pastagem predominantemente de Brachiaria brizantha (lotação de 0,8 a 1,0 UA/ha), sendo divididos em grupos contemporâneos (G1 e G2), em função da data de nascimento e do manejo, sendo o G1 os nascidos de 01/10 a 09/12/1996 e o G2 de 10/12/1996 a 28/02/1997. As temperaturas médias máximas e mínimas e o índice pluviométrico (chuvas), nos períodos em que ocorreram os nascimentos e a desmama do G1 e G2, respectivamente, estão apresentados na Tabela 1.

As mensurações testiculares foram realizadas na ocasião das pesagens, aos 12 meses $(375,09 \pm 22,01$ dias de idade, em média), nos meses de novembro 1997 (NOV/G1) e janeiro 1998 (JAN/G2), e aos 18 meses $(553,09 \pm 21,43$ dias, em média) nos meses de maio (MAI/G1) e julho (JUL/G2) 1998.

Os parâmetros observados foram a circunferência escrotal (CE), o comprimento (COMP), a largura (LARG) e o volume testicular (VOL). A CE foi medida por meio de fita métrica metálica na região mediana dos testículos e o COMP e a LARG, por paquímetro. Para medir o COMP, foram considerados os testículos, excluindo a cauda dos epidídimos no sentido dorso-ventral, e a LARG, tendo sido medida na região mediana de cada testículo no sentido latero-medial.

Inicialmente, para se obter a fórmula que melhor representasse o VOL em relação às medidas testiculares, foram feitas medições in vitro (testículos de abatedouros) e in situ (no animal), por intermédio de paquímetro e deslocamento de líquido. Constatou-se, por intermédio das medições in vitro, que não houve diferença significante entre os testículos direito e esquerdo, concordando com o relato de ALBARRAN et al. (1988). Para calcular o VOL, foi adotada a fórmula que mais se aproximou do valor obtido por deslocamento de líquido, a fórmula do cilindro, a qual também foi usada por FIELDS et al. (1979):

$$
\mathrm{VOL}=2\left[\left(\mathrm{r}^{2}\right) \times \Pi \mathrm{xh}\right]
$$

em que $r=$ raio calculado a partir da largura $(L A R G / 2)$, $\mathrm{h}=$ comprimento ou altura, $\mathrm{e} \Pi(\mathrm{Pi})=3,14$. O volume foi expresso em $\mathrm{cm}^{3}$ e representou os dois testículos.

Ainda foram considerados, para efeito de análise, os pesos vivos aos 12 e 18 meses de idade.

Para determinar a forma dos testículos, foi calculada a razão entre a sua largura e o comprimento (razão LARG/COMP), segundo BAILEY et al. (1996), na escala de 1 a 0,5 , em que 1 significa $\mathrm{LARG}=$ COMP e 0,5, LARG = 1/2 COMP. Em função desta escala, foram estabelecidas as seguintes formas:

razão $1 \leq 0,5=$ longo;

Tabela 1 - Médias de temperaturas máximas e mínimas e de chuvas na região de Brotas/SP durante a época de nascimento e desmama, em função dos grupos contemporâneos de machos da raça Nelore, PO

Table 1 - Means of maximum and minimum temperature and rainfall in Brotas/SP region during the birth and weaning season according to contemporary groups of Nellore straightbred males

\begin{tabular}{|c|c|c|c|c|c|c|}
\hline \multirow[t]{3}{*}{$\begin{array}{l}\text { Grupo contemporâneo } \\
\text { Contemporary group }\end{array}$} & \multicolumn{3}{|c|}{$\begin{array}{c}\text { Época dos nascimentos } \\
\text { Birth season }\end{array}$} & \multicolumn{3}{|c|}{$\begin{array}{c}\text { Época da desmama } \\
\text { Weaning season }\end{array}$} \\
\hline & \multicolumn{2}{|c|}{$\begin{array}{l}\text { Temperaturas médias }\left({ }^{\circ} \mathrm{C}\right) \\
\text { Mean temperature }\end{array}$} & \multirow[t]{2}{*}{$\begin{array}{l}\text { Chuvas }(\mathrm{mm}) \\
\text { Rainfall }(\mathrm{mm})\end{array}$} & \multicolumn{2}{|c|}{$\begin{array}{l}\text { Temperaturas médias }\left({ }^{\circ} \mathrm{C}\right) \\
\text { Mean temperature }\end{array}$} & \multirow[t]{2}{*}{$\begin{array}{l}\text { Chuvas }(\mathrm{mm}) \\
\text { Rainfall }(\mathrm{mm})\end{array}$} \\
\hline & $\begin{array}{l}\text { Máxima } \\
\text { Maximum }\end{array}$ & $\begin{array}{l}\text { Mínima } \\
\text { Minimum }\end{array}$ & & $\begin{array}{c}\text { Máxima } \\
\text { Maximum }\end{array}$ & $\begin{array}{l}\text { Mínima } \\
\text { Minimum }\end{array}$ & \\
\hline$\overline{\mathrm{G} 1}$ & $31,0-35,1$ & $12,4-15,3$ & $102-112$ & 30,6 & 11,0 & 74 \\
\hline G2 & $32,7-33,3$ & $13,7-17,1$ & $137-353$ & 32,4 & 11,2 & 6 \\
\hline
\end{tabular}


138 Rev. bras. zootec.

razão 2 de 0,51 a $0,625=$ longo/moderado;

razão 3 de 0,626 a $0,750=$ longo/oval;

razão 4 de 0,751 a $0,875=$ oval/esférico; e

razão $5>0,875=$ esférico.

Foi, ainda, calculado o crescimento testicular (CT) dos parâmetros circunferência escrotal (CT 12 a 18), comprimento (CC 12 a 18), largura (CL 12 a 18) e volume (VOL 12 a 18), por meio da diferença entre os valores observados aos 12 e 18 meses de idade.

As características utilizadas para análise estatística foram CE, COMP, LARG, VOL e razão LARG/ COMP, aos 12 e 18 meses de idade, e o crescimento testicular entre as idades consideradas.

Os resultados foram analisados pelo procedimento GLM do STATISTICAL ANALYSIS SYSTEM (SAS, 1990). O modelo matemático usado continha os efeitos fixos do grupo contemporâneo (G1 e G2), mês da coleta dos dados dentro de grupo (NOV, JAN, MAI, JUL) e da forma dos testículos (razão LARG/ COMP de 1 a 4). Os pesos aos 12 e 18 meses de idade foram incluídos como covariáveis. As correlações foram calculadas utilizando o procedimento CORR.

\section{Resultados e Discussão}

Os valores de CE, COMP, LARG e VOL e pesos aos 375 e 553 dias de idade, em média, ou seja 12 e 18 meses, respectivamente, estão apresentados na Tabela 2.
Os valores observados para CE e VOL, tanto aos 12 como 18 meses de idade, foram semelhantes aos descritos na literatura para a raça Nelore (SILVA, 1997; UNANIAN e SILVA, 1997).

A análise de variância destes dados revelou variação $(\mathrm{P}<0,0001)$, em função do grupo contemporâneo e peso. O mês de coleta de dados somente influenciou o VOL e a LARG aos 12 meses de idade.

As médias dos parâmetros que variaram em função da idade e do grupo contemporâneo estão apresentadas na Tabela 3.

Observou-se que os maiores valores de CE, VOL e LARG aos 12 meses de idade ocorreram nos machos do G1. Aos 18 meses, não houve diferença entre CE do G1 e G2. Nesta idade somente foram observadas variações de VOL, COMP e LARG, sendo as médias do VOL e COMP maiores no G1.

Estas variações, em função do grupo contemporâneo, dentro da mesma idade e do mês de coleta de dados, mostraram que estes parâmetros sofreram a influência da sazonalidade.

A sazonalidade - o efeito da nutrição e do clima (precipitação pluviométrica e temperatura) sobre o desenvolvimento testicular, principalmente a circunferência escrotal - foi amplamente descrita em ovinos (COLAS et al., 1987; LINDSAY et al., 1984) e bovinos (SILVA et al., 1991; GODFREY et al., 1990). A variação estacional representada pela disponibilida-

Tabela 2 - Médias ( $\pm D P)$, mínima e máxima de parâmetros testiculares e peso vivo, em machos da raça Nelore, PO Table 2 - Means $( \pm S D)$, minimum and maximum values of testicular parameters and the live weight, in Nellore straightbred males

\begin{tabular}{|c|c|c|c|c|c|c|}
\hline$\overline{\text { Variável }}$ & Animais & Idade (dias) & Médias & $\mathrm{DP}$ & Mínima & Máxima \\
\hline Trait & Animals & Age (days) & Means & $S D$ & Minimum & Maximum \\
\hline Circunferência escrotal $12(\mathrm{~mm})$ & 420 & $375,09 \pm 22,01$ & 184,89 & 15,79 & 146,00 & 240,00 \\
\hline $\begin{array}{l}\text { Scrotal circunference } 12(\mathrm{~mm}) \\
\text { Comprimento testicular } 12(\mathrm{~mm})\end{array}$ & & & 65,96 & 6,48 & 49,00 & 100,00 \\
\hline Testicular length $12(\mathrm{~mm})$ & & & & & & \\
\hline $\begin{array}{l}\text { Largura testicular } 12(\mathrm{~mm}) \\
\text { Testicular width } 12(\mathrm{~mm})\end{array}$ & & & 32,58 & 4,63 & 20,00 & 53,00 \\
\hline Volume testicular $12\left(\mathrm{~cm}^{3}\right)$ & & & 113,80 & 42,03 & 32,66 & 361,73 \\
\hline $\begin{array}{l}\text { Testicular volume } 12\left(\mathrm{~cm}^{3}\right) \\
\text { Peso } 12(\mathrm{~kg}) \\
\text { Weight } 12(\mathrm{~kg})\end{array}$ & 418 & & 240,23 & 26,64 & 170,00 & 311,00 \\
\hline $\begin{array}{l}\text { Circunferência escrotal } 18(\mathrm{~mm}) \\
\text { Scrotal circunference } 18(\mathrm{~mm})\end{array}$ & 402 & $553,09 \pm 21,43$ & 236,81 & 22,36 & 180,00 & 305,00 \\
\hline Comprimento testicular $18(\mathrm{~mm})$ & & & 79,31 & 11,16 & 40,00 & 140,00 \\
\hline $\begin{array}{l}\text { Testicular length } 18(\mathrm{~mm}) \\
\text { Largura testicular } 18(\mathrm{~mm})\end{array}$ & & & 42,92 & 5,83 & 25,00 & 60,00 \\
\hline $\begin{array}{l}\text { Testicular width } 18(\mathrm{~mm}) \\
\text { Volume testicular } 18\left(\mathrm{~cm}^{3}\right) \\
\text { Testicular volume } 18\left(\mathrm{~cm}^{3}\right)\end{array}$ & & & 239,26 & 90,56 & 65,74 & 714.13 \\
\hline $\begin{array}{l}\text { Peso } 18(\mathrm{~kg}) \\
\text { Weight } 18(\mathrm{~kg})\end{array}$ & 387 & & 308,06 & 36,42 & 213,00 & 409,00 \\
\hline
\end{tabular}


UNANIAN et al.

Tabela 3 - Médias ( \pm erro-padrão) de parâmetros testiculares, em função da idade e do grupo contemporâneo, em machos da raça Nelore, $\mathrm{PO}$

Table 3 - Means ( \pm standard error) of testicular parameters according age and contemporary group in straightbred Nellore males

\begin{tabular}{|c|c|c|c|c|c|c|}
\hline $\begin{array}{l}\text { Grupo } \\
\text { contemporâneo } \\
\text { Contemporary } \\
\text { group }\end{array}$ & $\begin{array}{l}\text { Animal (dias) } \\
\text { Animal (days) }\end{array}$ & $\begin{array}{l}\text { Idade (dias) } \\
\text { Age (days) }\end{array}$ & $\begin{array}{l}\text { Circunferência } \\
\text { escrotal (mm) } \\
\text { Scrotal } \\
\text { circunference }\end{array}$ & $\begin{array}{c}\text { Comprimento } \\
\text { testicular (mm) } \\
\text { Testicular } \\
\text { length } \\
\end{array}$ & $\begin{array}{c}\text { Largura } \\
\text { testicular }(\mathrm{mm}) \\
\text { Testicular } \\
\text { Width }\end{array}$ & $\begin{array}{c}\text { Volume } \\
\text { testicular }\left(\mathrm{cm}^{3}\right) \\
\text { Testicular } \\
\text { volume }\end{array}$ \\
\hline G1 & 208 & $361,78 \pm 1,02$ & $186,59 \pm 0,97^{a}$ & $65,70 \pm 0,40^{\mathrm{a}}$ & $33,02 \pm 0,30^{\mathrm{a}}$ & $115,58 \pm 2,53^{a}$ \\
\hline $\mathrm{G} 2$ & 210 & $388,29 \pm 1,32$ & $182,84 \pm 1,15^{b}$ & $66,01 \pm 0,46^{b}$ & $32,07 \pm 0,33^{b}$ & $110.83 \pm 3,02^{b}$ \\
\hline G1 & 188 & $540,78 \pm 1,02$ & $243,83 \pm 1,62$ & $80,13 \pm 0,91^{\mathrm{a}}$ & $42,67 \pm 0,47^{a}$ & $241,33 \pm 7,43^{\mathrm{a}}$ \\
\hline $\mathrm{G} 2$ & 199 & $565,29 \pm 1,32$ & $230,33 \pm 1,45$ & $78,56 \pm 0,69^{b}$ & $43,29 \pm 0,36^{b}$ & $238,63 \pm 5,59^{b}$ \\
\hline
\end{tabular}

Médias, dentro de cada faixa etária, seguidas de letras diferentes são diferentes $(P<0,0001)$.

Means within each age followed by different letters are different $(P<.0001)$

de alimentar influi no crescimento e nas dimensões testiculares, como observado por LINDSAY et al (1984) em ovinos e SILVA et al. (1991) em bovinos.

Apesar de os animais deste estudo terem nascido no período chuvoso, existiu diferença de dois a seis meses entre os nascimentos, que proporcionou aos nascidos do G1 período de disponibilidade de alimentos mais longo. Este mesmo grupo teve melhores condições climáticas por ocasião da desmama. O sistema de criação, por ter sido exclusivamente a pasto, pode sofrer mudanças mesmo dentro da mesma época, em função do diferente regime de chuvas e, conseqüentemente, a qualidade alimentar pode não ser a mesma. Este fato determinou a diferença no desenvolvimento testicular observada entre os grupos, ao contrário do relatado por VIEIRA et al.(1988) em animais suplementados.

Entre as características estudadas, o VOL apresentou valores médios aos 12 meses de idade superiores aos relatados por ALENCAR e VIEIRA (1989) de $56,6 \mathrm{~cm}^{3}$, em machos da raça Canchim, e NWAKALOR e OBASI (1991) de $189,9 \mathrm{~cm}^{3}$, em bovinos N'Dama. Aos 18 meses, os valores médios do volume continuaram mais altos que os obtidos por ALENCAR e VIEIRA (1989) de 107,8 $\mathrm{cm}^{3}$, sendo, no entanto, semelhantes aos de BAILEY et al. (1998), de $260,00 \mathrm{~cm}^{3}$, encontrados em touros adultos sem raça definida (touros de abatedouros). Ainda aos 18 meses, o VOL médio dos machos da raça Nelore foi inferior ao observado por GODFREY et al. (1990), de 435,7 $\mathrm{cm}^{3}$, em animais da raça Brahman aos 19 meses de idade. Contudo, ressalta-se que a fórmula matemática adotada para cálculo de VOL, neste estudo, foi diferente da descrita por BAILEY et al. (1998), porém semelhante à de FIELDS et al. (1979).

A medição do VOL, neste trabalho, baseou-se nas características morfológicas dos zebuínos que, normalmente, possuem testículos mais longos do que os taurinos, motivo pelo qual apresentam CE menores (SILVA et al., 1991). Ainda, a LARG e o COMP dos testículos dos machos da raça Nelore foram menores aos descritos por BAILEY et al. (1998), de 66 a $70 \mathrm{~mm}$ e 118 a $140 \mathrm{~mm}$, respectivamente, que aplicaram a fórmula do elipsóide para medir o volume testicular em animais da raça Holstein, assim como ALENCAR e VIEIRA (1989), em animais da raça Canchim.

Para testar a fórmula que melhor representasse o volume testicular dos machos Nelore, foram feitas, além de medidas in situ, medidas in vitro (medidas de testículos pós-morte por deslocamento de líquido e por paquímetro). Estas medidas mostraram que a fórmula do cilindro é a mais adequada à morfologia dos testículos do Nelore, principalmente aos 12 meses de idade. A inviabilidade da fórmula do elipsóide foi também relatada por YADAV e SHARMA (1994), os quais constataram que a mesma superestimava o volume testicular.

Apesar de não existir concordância quanto à fórmula matemática para medição do volume testicular, provavelmente em função da variação do tipo dos testículos entre as raças bovinas, segundo BAILEY et al. (1998), o volume testicular pode ser medido de forma precisa, desde que se utilizem medidas bidimensionais, como o comprimento e a largura dos testículos.

Considerando todos os parâmetros testiculares do estudo, verificou-se que suas correlações foram positivas e altamente significantes $(\mathrm{P}<0,0001)$ dentro e entre os grupos contemporâneos (Tabela 4).

O VOL aos 12 e 18 meses de idade mostrou-se altamente correlacionado com a $\mathrm{CE}$ aos 12 e 18 meses, sendo ainda maiores as correlações da LARG e do COMP com o VOL, tanto aos 12 como 18 meses de idade. Todos os parâmetros estudados correlacionaram com os pesos.

As correlações obtidas foram semelhantes às descritas na literatura para bovinos de corte e leite. A 
140 Rev. bras. zootec.

correlação do VOL com LARG foi superior à observada por ROCHA et al. (1990), de 0,78, em animais Santa Gertrudis aos 16,5 meses, e a do VOL com a $\mathrm{CE}$ foi semelhante à observada por MOHANTY et al. (1991) de 0,64, em machos da raça Holstein. As correlações da CE com o peso foram mais baixas que as observadas por ALENCAR e VIEIRA (1989) de 0,85, em animais Canchim, e semelhantes às do VOL $(0,52)$ com o peso. Ainda, estas correlações foram mais baixas que as descritas por BARBA (1985) e SILVA et al. (1991) em machos zebu, de 0,85 e 0,92, respectivamente.

Embora as correlações tenham sido altamente significativas $(\mathrm{P}<0,0001)$, observou-se que alguns dos parâmetros testiculares apresentaram coeficientes maiores que os outros. A alta correlação observada entre CE e o VOL aos 12 e 18 meses de idade mostra a possibilidade de se avaliar e predizer o potencial reprodutivo, por ocasião da seleção de machos para reprodutores, utilizando-se qualquer um destes parâmetros. Ainda, observando a alta correlação existente entre o CE 12 e 18, e mesmo entre os VOL nestas idades, é possível afirmar que a escolha de machos, em função destas características, pode ser realizada aos 12 meses.

A forma testicular dos machos da raça Nelore, obtida pela distribuição dos animais, em função da razão LARG/COMP, revelou a existência de apenas quatro dos cinco grupos inicialmente descritos. Não foram encontrados animais com testículos esféricos (razão > 0,875), sendo que a forma oval/esférica (razão 0,751 a 0,875) apareceu somente em um animal aos 12 meses e dois animais aos 18 meses de idade (Tabela 5). O teste Qui-quadrado entre as freqüências mostrou diferença $(\mathrm{P}<0,05)$ entre as formas aos 12 e 18 meses de idade, representada pela razão LARG/COMP.

A forma testicular obtida em função da razão LARG/COMP revelou que os machos deste estudo, na sua maioria $(87 \%)$, possuíam testículos longos (razão $\leq 0,5$ ) e longos moderados (razão 0,51 a 0,625), tanto aos 12 como 18 meses de idade. Considerando as formas individualmente, houve diminuição de $46 \%$ no número de animais com testículos longos e aumento de $55 \%$ na freqüência de longos moderados, tendo ocorrido, porém, o maior aumento no grupo dos animais com testículos longos/ovais (razão 0,626 a 0,750). Estes resultados mostraram que, apesar da predominância das formas testiculares longas, as mesmas mudaram com a idade (Figura 1), ou seja, em $73 \%$ dos animais os testículos ficaram mais ovais.

A CE, o COMP, a LARG e o VOL, aos 12 meses de idade, variaram em função da forma dos testículos (razão LARG/COMP). Aos 18 meses, porém, esta variação ocorreu somente no COMP e na LARG, não havendo diferença do VOL e da CE entre as formas testiculares (Tabela 6).

Pelos resultados obtidos aos 18 meses de idade, a ausência de diferença entre os valores da CE e do VOL deve ter ocorrido em função da predominância

Tabela 4 - Correlações entre os parâmetros testiculares em machos da raça Nelore, PO

Table 4 - Correlations between testicular parameters in straightbred Nellore males

\begin{tabular}{|c|c|c|c|c|c|c|c|c|c|}
\hline & COMP 12 & LARG 12 & VOL 12 & CE 18 & COMP 18 & LARG 18 & VOL 18 & Peso 12 & Peso 18 \\
\hline CE 12 & 0,67704 & 0,73506 & 0,78023 & 0,70584 & 0,46695 & 0,73506 & 0,59592 & 0,41585 & 0,46099 \\
\hline COMP12 & & 0,52359 & 0,71638 & 0,52849 & 0,51922 & 0,46306 & 0,51753 & 0,33671 & 0,29397 \\
\hline LARG 12 & & & 0,95027 & 0,56917 & 0,37866 & 0,48097 & 0,49684 & 0,34602 & 0,37840 \\
\hline VOL 12 & & & & 0,60541 & 0,44749 & 0,51893 & 0,55283 & 0,37100 & 0,37967 \\
\hline CE 18 & & & & & 0,72787 & 0,74090 & 0,80096 & 0,34078 & 0,61765 \\
\hline COMP18 & & & & & & 0,63222 & 0,82467 & 0,34592 & 0,39955 \\
\hline LARG 18 & & & & & & & 0,93734 & 0,41651 & 0,31543 \\
\hline VOL 18 & & & & & & & & 0,41010 & 0,38633 \\
\hline
\end{tabular}

Nível de significância das correlações: $P<0,0001$.

Level of significance: $P<.001$.

Número de animais: 373 a 420 .

Animals number: 373 to 420 .

CE 12 e 18 = Circunferência escrotal, $\mathrm{mm}$, aos 12 e 18 meses de idade.

CE 12 and $18=$ Scrotal circunference, $\mathrm{mm}$, at 12 and 18 months of age.

COMP 12 e 18 = Comprimento testicular, $\mathrm{mm}$, aos 12 e 18 meses de idade.

COMP 12 and $18=$ Testicular length, $\mathrm{mm}$, at 12 and 18 months of age.

LARG 12 e 18 = Largura testicular, $\mathrm{mm}$, aos 12 e 18 meses de idade.

LARG 12 and $18=$ Testicular width, $\mathrm{mm}$, at 12 and 18 months of age.

VOL 12 e $18=$ Volume testicular, $\mathrm{cm}^{3}$, aos 12 e 18 meses de idade.

VOL 12 and $18=$ Testicular volume, $\mathrm{cm}^{3}$, at 12 and 18 months of age. 
das formas testiculares alongadas (87\% de forma longa). Como não foram realizadas coletas de sêmen, que eventualmente poderiam estabelecer algumas diferenças, foi difícil avaliar a influência dessas formas sobre o desempenho reprodutivo destes animais.

Houve, ainda, correlação entre a forma testicular (razão LARG/COMP), aos 12 meses, e a CE e o $\operatorname{VOL}(0,30$ e 0,50 , respectivamente; $\mathrm{P}<0,0001)$. Aos 18 meses, esta correlação ocorreu apenas com o VOL $(0,12 ; \mathrm{P}<0,05)$.

Considerando o crescimento dos testículos (CE, COMP, LARG e VOL), em relação à forma testicular, foi observado aumento significante da largura testicular (CL) (Tabela 7).

A CL de 12 a 18 meses foi maior nos testículos longos, não sendo constatadas diferenças, nos demais parâmetros, para crescimento entre as formas testiculares. Esta observação, além daquela de aumento do número de animais com testículos longos moderados e longos/ovais, também evidenciou a mudança na forma testicular.

Entretanto, a mudança na forma testicular que ocorreu dos 12 aos 18 meses de idade, deve ser considerada com reserva, sendo necessários mais estudos para sua comprovação. Nem mesmo BAILEY et al. (1998), que estudaram a forma testicular em função da sua importância na produção de espermatozóides, não relataram este fato. Esses autores apenas observaram que houve variação entre as formas testiculares, a qual foi responsável pela diferença na produção espermática. No entanto, as mu-

Tabela 5 - Freqüências (\%) das formas dos testículos (razão LARG/COMP) em função da idade, em machos da raça Nelore, $P O$

Table 5 - Frequencies of testicular shape (LARG/COMP ratio) by age in straightbred Nellore males

\begin{tabular}{|c|c|c|}
\hline \multirow[t]{2}{*}{$\begin{array}{l}\text { Forma do testículo } \\
\text { Testicular shape }\end{array}$} & \multicolumn{2}{|c|}{$\begin{array}{l}\text { Freqüência }(\%) \\
\text { Frequency }\end{array}$} \\
\hline & 12 meses & 18 meses \\
\hline Longa (razão $\leq$ 0,5) & $58,81^{\mathrm{a}}$ & $26,87^{b}$ \\
\hline Long $($ ratio $\leq .5)$ & & \\
\hline $\begin{array}{l}\text { Longa moderada (razão } 0,51 \text { a } 0,625) \\
\text { Long moderate (ratio } .51 \text { to } .625 \text { ) }\end{array}$ & $38,81^{\mathrm{a}}$ & $60,19^{b}$ \\
\hline $\begin{array}{l}\text { Longa/oval (razão } 0,626 \text { a } 0,750 \text { ) } \\
\text { Long/ovoid (ratio .626 to .750) }\end{array}$ & $2,14^{\mathrm{a}}$ & $12,69^{b}$ \\
\hline $\begin{array}{l}\text { Oval/esférica (razão } 0,751 \text { a } 0,875 \text { ) } \\
\text { Ovoid/spheroid (ratio } .751 \text { to } .875 \text { ) }\end{array}$ & 0,24 & 0,25 \\
\hline $\begin{array}{l}\text { Esférica (razão }>0,875 \text { ) } \\
\text { Spheroid (ratio }>.875 \text { ) }\end{array}$ & 0 & 0 \\
\hline
\end{tabular}

Médias, na mesma linha, seguidas de letras diferentes, são diferentes $(P<0,05)$ pelo teste Qui-quadrado.

Means, within a row, followed by different letters are different $(P<.05)$ by $\chi^{2}$ test. Meses (months). danças na forma testicular, com a idade, provavelmente ocorrem, pois, aos 12 meses de idade, os animais encontram-se em desenvolvimento, os testículos crescem tanto em largura como comprimento. Caso a mudança na forma testicular, neste período, seja comprovada, é preciso que a seleção de machos para reprodutores, apenas pela circunferência escrotal, seja feita com reservas. Considerando-se apenas estes parâmetros, corre-se o risco de eliminar animais, que na fase adulta poderiam ser utilizados na reprodução. Convém lembrar que, dos 12 aos 20 a 24 meses de idade, conforme mostrado por FIELDS et al. (1979) em animais Brahman, o crescimento testicular ocorre de forma muito rápida e, quando alcançam 24 meses, os seus tamanhos assemelham-se aos dos machos taurinos. Estas observações foram feitas tanto para a circunferência escrotal como, principalmente, para o volume testicular, o qual tem sido apontado como medida necessária na seleção de reprodutores.

A predominância da forma testicular longa, característica de zebuínos, em particular a raça Nelore, em função da circunferência escrotal normalmente pequena, tem preocupado os criadores ao selecionarem os seus reprodutores. Este fato deve-se, em boa parte, aos critérios adotados na seleção pelo tamanho da circunferência escrotal, em que machos com testículos longos podem ser eliminados por apresentarem circunferências menores que os seus contemporâneos de testículos ovais (SILVA et al., 1991).

O conceito referente aos testículos longos despertou o interesse por pesquisas (BAILEY et al., 1996 e 1998) para verificar se a forma longa realmente prejudica o potencial reprodutivo do macho. Os autores mostraram que ejaculados provenientes de testículos longos apresentaram maior concentração de espermatozoides/mL que os testículos ovóides, embora estes últimos apresentassem circunferências maiores. Segundo esses autores, a maior concentração de espermatozóides/mL deve-se à melhor termoregulação, oriunda de melhor distribuição de vasos sangüíneos e túbulos seminíferos, importantes na produção espermática. Estas pesquisas concluíram que somente a circunferência escrotal não é suficiente para predizer a produção espermática, a qual está ligada a outros fatores, como o volume testicular, além da forma dos testículos.

A circunferência escrotal tem sido um parâmetro valioso na seleção de reprodutores, por indicar o potencial de produção espermática diária, além de ser uma característica altamente herdável e apresentar correlação com outros parâmetros testiculares além do peso. No entanto, BERTSCHINGER et al. (1992) 
a.

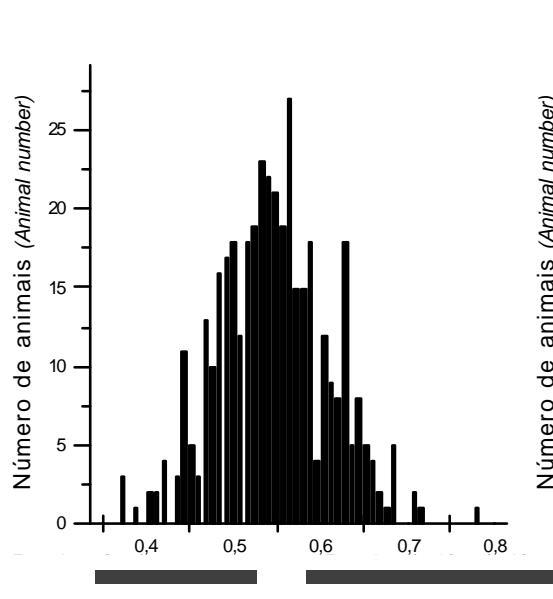

Razão LARG/COMP aos 12 meses de idade LARG/COMP ratio at 12 months of age b.

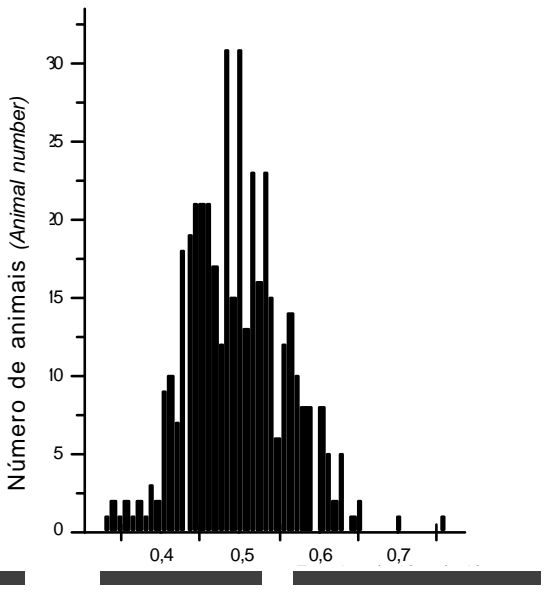

Razão LARG/COMP aos 18 meses de idade LARG/COMP ratio at 18 months of age c.

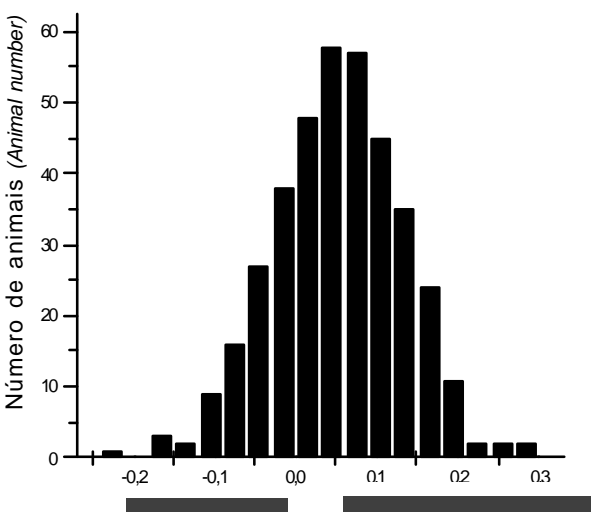

Mudança da forma testicular dos 12 aos 18 meses de idade Testicular shape change from 12 to 18 months of age

Figura 1 - Distribuição da freqüência das formas testiculares: a. aos 12 meses de idade; b. aos 18 meses de idade; c. mudança entre as formas testiculares em função da idade, em machos de raça Nelore, PO.

Figure 1 - Frequencies of testicular shape: a. at 12 month of age; b. at 18 month of age; $c$. testicular shape changes according to age, in Nelore straightbred males.

Tabela 6 - Médias ( \pm erro-padrão) da circunferência escrotal (CE/mm) e volume testicular (VOL/cm $\left.{ }^{3}\right)$ aos 12 e 18 meses de idade em função da forma do testículo (razão LARG/COMP), em machos da raça Nelore, PO

Table 6 - Means ( \pm standard error) of scrotal circunference $(\mathrm{CE} / \mathrm{mm})$ and testicular volume $\left(\mathrm{VOL} / \mathrm{cm}^{3}\right)$ at 12 and 18 months of age according to testicular shape (LARG/COMP ratio) in Nellore straightbred males

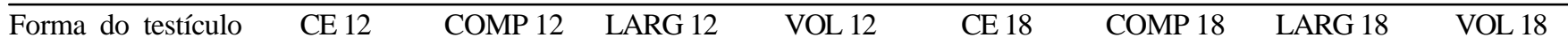
Testicular shape

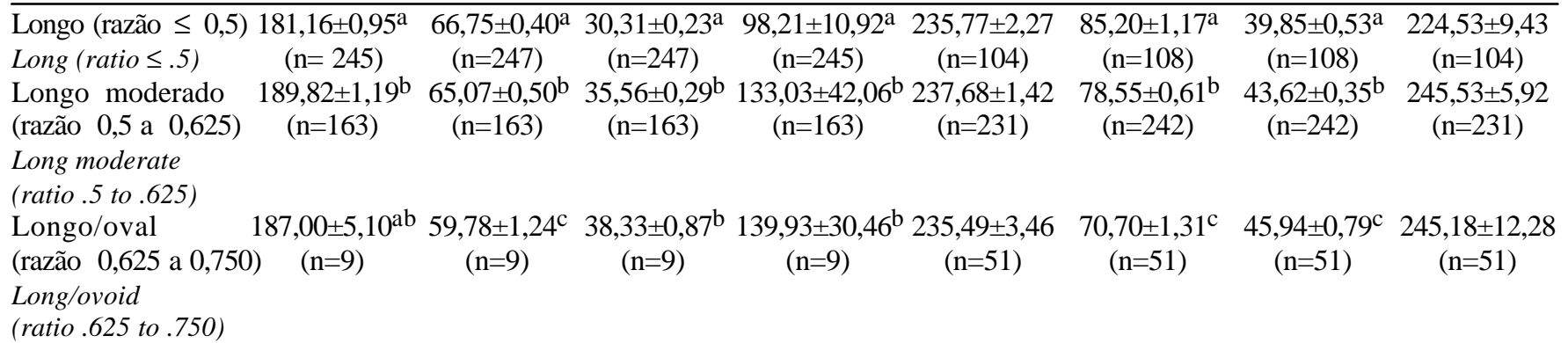

Médias, na coluna, seguidas de letras diferentes são significantes a $5 \%$.

Para as abreviações, veja definições na Tabela 4.

Means within a column followed by different letters are significant at $5 \%$ level.

For the definition of the abbreviations see Table 4. 
UNANIAN et al.

Tabela 7 - Médias ( \pm erro-padrão) do crescimento testicular dos 12 aos 18 meses de idade, em função da forma do testículo (razão LARG/COMP), em machos Nelore, PO

Table 7 - Means ( \pm standard error) of testiculargrowth from 12 to 18 months of age according to testicular shape (LARG/ COMP ratio) in Nellore straightbred males

\begin{tabular}{|c|c|c|c|c|c|}
\hline $\begin{array}{l}\text { Forma dos testículos } \\
\text { Testicular shape }\end{array}$ & $\begin{array}{l}\text { Animal } \\
\text { Animal }\end{array}$ & CT 12 a 18 & CC 12 a 18 & CL 12 a 18 & VOL 12 a 18 \\
\hline $\begin{array}{l}\text { Longo }(\text { razão } \leq 0,5) \\
\text { Long }(\text { ratio } \leq .5)\end{array}$ & 224 & $52,26 \pm 1,02$ & $12,58 \pm 0,61$ & $12,21 \pm 0,31^{\mathrm{a}}$ & $134,05 \pm 4,61$ \\
\hline $\begin{array}{l}\text { Longo moderado (razão } 0,51 \text { a } 0,625) \\
\text { Long moderate (ratio } .51 \text { to } .625 \text { ) }\end{array}$ & 148 & $51,78 \pm 1,32$ & $14,52 \pm 0,73$ & $8,21 \pm 0,41^{b}$ & $116,63 \pm 2,01$ \\
\hline Longo/oval (razão 0,626 a 0,750 ) & 6 & $47,17 \pm 6,70$ & $14,00 \pm 4,00$ & $5,17 \pm 2,61^{b}$ & $92,47 \pm 36,63$ \\
\hline
\end{tabular}

Long/ovoid (ratio .626 to .750)

Médias, na coluna, seguidas de letras diferentes, são significantes em nível de $5 \%$.

CT 12 a 18 = Crescimento da circunferência escrotal de 12 aos 18 meses de idade $(\mathrm{mm})$.

CC 12 a $18=$ Crescimento do comprimento dos testículos de 12 aos 18 meses de idade $(\mathrm{mm})$.

CL 12 a $18=$ Crescimento da largura dos testículos de 12 aos 18 meses de idade $(\mathrm{mm})$.

VOL 12 a 18 = Crescimento do volume dos testículos de 12 aos 18 meses de idade ( $\left.\mathrm{cm}^{3}\right)$.

Means, within a column, followed by different letters are significant at $5 \%$ level.

CT 12 to 18 = Scrotal circunference growth from 12 to 18 months of age $(\mathrm{mm})$

CC 12 to 18 = Testicular length growth from 12 to 18 months of age $(\mathrm{mm})$.

CL 12 to 18 = Testicular width growth from 12 to 18 months of age $(\mathrm{mm})$.

VOL 12 to 18 = Testicular volume growth from 12 to 18 months of age (cni)

verificaram, principalmente em animais jovens, que machos com alta circunferência escrotal podem apresentar ejaculados com baixa motilidade e elevada porcentagem de espermatozóides anormais.

\section{Conclusões}

Em função da forma mais alongada dos testículos de zebuínos e, ainda, das observações deste estudo, concluiu-se que, ao se praticar seleção em animais jovens, deve-se utilizar, além da circunferência escrotal, o volume testicular. Estes parâmetros associados devem avaliar com maior precisão o potencial do futuro reprodutor.

Quanto à forma testicular, apesar de o número de animais deste estudo ter sido grande, acredita-se que, para demonstrar a importância morfo-fisiológica desta característica, torna-se necessário realizar pesquisas adicionais para confirmar os resultados encontrados neste trabalho e, ainda, estabelecer critérios para identificação desta característica.

\section{Agradecimento}

Ao médico veterinário Magno Roberto de Souza da Fazenda Mundo Novo, pela colaboração na condução do trabalho.

\section{Referências Bibliográficas}

ALBARRAN, I., BIDOT, A., GONZALES, J.W. 1988. Estructura corporal y dinamica testicular de los machos 5/ 8 Holstein - 3/8 Cebu y Siboney de Cuba de 0 a 12 meses de edad. Rev. Cubana Cienc. Vet., 19(1):65-71.
ALENCAR, M.M., VIEIRA, R.C. 1989. Crescimento testicular de touros da raça Canchim. Pesq. Agopec. Bras., 24(11):1329-1333.

BAILEY, T.L., HUDSON, R.S., POWE, T.A. et al. 1998. Caliper and ultrasonographic measurements of bovine testicles and a mathematical formula for determining testicular volume and weight in vivo. Theriogenology, 49(10):581-598.

BAILEY, T.L., MONKE, D., HUDSON, R.S. et al. 1996. Testicular shape and its relationship to sperm production in mature Holstein bulls. Theriogenology, 46(3):881-887.

BARBA, F. 1985. Estudio clinico de los organos genitales de touros Cebu en monta directa. Rev. Cubana Reprod. Anim., 11(1):23-35.

BERTSCHINGER, H.J., EHRET, W.J., WOOD, R. et al. Beef bull performance, scrotal circumference and semen quality. In: INTERNATIONAL CONGRESS ON ANIMAL REPRODUCTION, 12, 1992. Hague. Proceedings...[s.1.: s.n.], 1992. v.3, p.1525-1527.

COLAS, G., GUERRIN, Y., BRIOIS, M. et al. 1987. Photoperiodic control of testicular growth in the ram lamb. Anim. Reprod. Sci., 13(4):255-262.

FIELDS, M.J., BURNS, W.C., WARNICK, A.C. 1979. Age, season and breed effects on testicular volume and semen traits in young beef bulls. J. Anim. Sci., 48(6):1299-1304.

LINDSAY, D.R., PELlETIER, J., PISSELET, C. et al. 1984. Changes in photoperiod and nutrition and their effect on testicular growth of rams. J. Reprod. Fert., 71(2):351-356.

MOHANTY, D.N., DUGWEKAR, Y.G., BISWAS, R.K. 1991. Testicular measurements of Holstein Friesian bulls as an indicator of reproductive performance. Orissa Vet. J, $16(1-2): 17-23$.

NWAKALOR, L.N., OBASI, U.O. 1991. Relationships of testicular size, body measurements and age in White Fulani (Bunaji) and N'Dama bulls. East African Agr. Forest. J., 57(1):23-31.

ROCHA, A., FORREST, D.W., CARPENTER, B.B. et al. 1990 Relationships among seminal vesicle size, testicular dimensions and copulatory activity in Santa Gertrudis bulls. In: Beef cattle research in Texas. College Station, USA: Texas Agricultural Experiment Station. p.185-188.

SAS INSTITUTE INC. 1990. SAS user's guide: statistics. 
144 Rev. bras. zootec.

Cary, NC. 956p.

SCHOEMAN, S.J., COMBRINK, G.C. Testicular growth patterns in three South African sheep breeds. In: WORLD CONGRESS ON GENETICS APPLIED TO LIVESTOCK PRODUCTION, 3, Lincoln, USA, 1986. Proceedings...Lincoln, USA: 1986. p.306-311.

SILVA, A.E.D.F. A identificação da puberdade, através do sêmen, em gado Nelore. In: SIMPÓSIO: O NELORE DO SÉCULO XXI, 4, Uberaba, MG, 1997. Anais... Uberaba, MG: 1997. p.51-58.

SILVA, A.E.D.F., DODE, M.A., PORTO, J.A. et al. 1991. Estacionalidade na atividade sexual de machos Nelore e mestiços Fleckvieh e Chianina x Nelore: características biométricas testiculares. Pesq. Agropec. Bras., 26(10):1745-1750.

UNANIAN, M., SILVA, A.E.D.F. Estudo da precocidade sexual em bovinos machos da raça Nelore. In: DIA de campo: 10/10/ 97. O melhoramento do Nelore a campo. \{s.1.\}: Fazenda Novo Mundo, Grupo Manah, 1997. p.6-8.
VIEIRA, R.C., ALENCAR, M.M., ESTEVES, S.N. 1988. Efeito da suplementação alimentar sobre o comportamento reprodutivo de tourinhos Canchim. Pesq. Agropec. Bras., 23(1):97-102.

YADAV, S.K., SHARMA, A.K. 1994. Seminiferous tubule length in normal buffalo-bulls and bucks. Int. J. Anim. Sci., 9(2):293-296.
Recebido em: 11/03/99

Aceito em: 04/06/99 